\title{
Influence of different biological environments on the stability of serotonin detection on carbon-based electrodes
}

\author{
Aidan Fagan-Murphy, Fiona Watt, Kelly A. Morgan \& Bhavik Anil Patel ${ }^{\star}$ \\ School of Pharmacy and Biomolecular Sciences, University of Brighton, Brighton, \\ BN2 4GJ
}

*Address for correspondence: Bhavik Anil Patel, School of Pharmacy and Biomolecular Sciences, University of Brighton, Brighton, BN2 4GJ, Tel.: +44 (0)1273 642418, Fax: +44

(0)1273 672674, Email: b.a.patel@brighton.ac.uk

\begin{abstract}
Serotonin $(5-\mathrm{HT})$ is a key neurotransmitter that is found in the brain, blood and gastrointestinal tract. Due to the interest in measuring this important biogenic amine in these various environments, measurements have been conducted using carbonbased sensors without understanding the influence of the matrix on the stability of recordings. Electrochemical recordings were carried out in PBS buffer, $0.5 \% \mathrm{w} / \mathrm{v}$ mucin and $5 \% \mathrm{w} / \mathrm{v}$ albumin on the glassy carbon (GC) and boron-doped diamond (BDD) electrodes. During recordings of $5-\mathrm{HT}$ on the GC electrode, the $5 \% \mathrm{w} / \mathrm{v}$ albumin matrix was protective against electrode fouling compared to $0.5 \% \mathrm{w} / \mathrm{v}$ mucin, which enhanced the rate of electrode fouling. On the BDD electrode, $0.5 \%$ $\mathrm{w} / \mathrm{v}$ mucin once again enhanced the rate of electrode fouling, however $5 \% \mathrm{w} / \mathrm{v}$ albumin did not alter the rate of fouling compared to PBS buffer. This data suggests that all proteins cannot be considered to behave in a similar fashion for electroanalytical measurements and thus careful consideration on the matrix effect needs to be considered before biological monitoring.
\end{abstract}

\section{Keywords}

Boron-doped diamond, glassy carbon, serotonin, fouling, biofouling 


\section{Introduction}

Serotonin (5-HT) is an important neurotransmitter that plays important biological roles in various areas of the body. $5-\mathrm{HT}$ is known to influence key neurological traits, such as anxiety [1], but also plays a key role in driving gastrointestinal motility and platelet aggregation [2, 3]. Within the brain $5-\mathrm{HT}$ is released in a microenvironment that contains large varying concentrations of key ions such as calcium, potassium and sodium. Studies have shown that the analytical current can be altered significantly in the presence of different concentrations of ions $[4,5]$. The majority of $5-\mathrm{HT}$ is found within the gastrointestinal (GI) tract. In the GI tract, $5-\mathrm{HT}$ is released from enterochromaffin cells that are located in the mucosa. Thus $5-\mathrm{HT}$ can be monitored in the lumen [6,7], where large quantities of mucin are also are present. Mucin is a glucoprotien that is released into the lumen by goblet cells to lubricate the bowel, which aids in the passage of food and waste products. The last location where $5-\mathrm{HT}$ is found is in the cardiovascular system, where it is present within platelets and released in conjunction with histamine to drive platelet aggregation. The blood is a very complex matrix that contains a large amount of biological proteins of which albumin is by far the most abundant.

Due to the diverse and important role 5-HT plays, it has been subject to various electroanalytical measurements. However, such measurements have been influenced by the degree of electrode fouling over time due to $5-\mathrm{HT}$ itself, as oxidative by-products are known to reduce electrode sensitivity. Another issue associated with biological measurements is the problem of bio-fouling, where matrix components, such as proteins have been shown to block the electrode surface over time. This is a particularly significant problem in the blood, where researchers have shown that sensors or devices implanted within the blood are subject to a hostresponse and thus results in the sensor being encapsulated as the biological system identifies it as a foreign body [8].

The majority of measurements of 5-HT have been conducted using carbon-based electrodes. Traditionally these recordings have been carried out on carbon fibre electrodes [9-11]; however boron-doped diamond [7, 12] and carbon nanotube electrodes $[13,14]$ have recently been shown to overcome electrode fouling from oxidative by-products. However limited insight is given on the influence of the 
background matrix towards the stability and sensitivity of $5-\mathrm{HT}$ measurements during these studies. It has been assumed that if a particular electrode performs well in one matrix, then it will also be suitable for recordings in another biological matrix.

This work examines the effect of different biological matrices on the response of 5HT on two commonly used carbon-based electrodes. Matrices that mimic the locations where $5-\mathrm{HT}$ is known to occur were employed during this study. The performance of these electrodes over an extended period of time will be discussed in the various matrices as well as the implications this may have on using similar sensors across different biological environments.

\section{Materials and Methods}

\subsection{Chemicals}

All the chemicals were purchased from Sigma-Aldrich and used without further purification. Deionised (DI) water was purified with a Millipore system and then used for rinsing equipment. 5-Hydroxytryptamine (5-HT/Serotonin), potassium ferrocyanide $(\mathrm{FeCN})$, potassium chloride and phosphate buffer saline (PBS) were all purchased from Sigma-Aldrich and used as received. To produce a matrix that mimics blood, $5 \% \mathrm{w} / \mathrm{v}$ bovine serum albumin in $0.1 \mathrm{M}$ PBS was used. Likewise to mimic the gastrointestinal lumen environment, $0.5 \% \mathrm{w} / \mathrm{v}$ of porcine mucin was employed. All solutions were adjusted to $\mathrm{pH} 7.4$

\subsection{Experimental set-up}

All electrochemical experiments were carried out using a $\mathrm{CHI}$ 630B potentiostat $(\mathrm{CHI}$ Instruments, Austin, Texas, USA). A three electrode setup was employed for all electrochemical studies using a platinum wire counter electrode and an $\mathrm{Ag} \mid \mathrm{AgCl}(3 \mathrm{M}$ $\mathrm{KCl})$ reference electrode $(\mathrm{CHI}$ Instruments, Austin, Texas, USA). The working electrode was either a boron-doped diamond (BDD) electrode (Winsor scientific LTD, diameter $3 \mathrm{~mm}$, boron doping level: $\approx 0.1 \%$, resistivity: $7.5 \times 10-4 \Omega \mathrm{m}$ ) or a glassy carbon (GC) electrode ( $\mathrm{CHI}$ Instruments, Austin, Texas, USA, diameter $3 \mathrm{~mm}$ ). Prior to electrochemical studies, the GC and BDD electrodes were polished for 5 minutes 
with $0.05 \mu \mathrm{m}$ grade alumina aqueous slurry; this procedure was carried out before each experimental run.

\subsection{Electrochemical protocol to study fouling}

In order to test the electrode prior to its use, a cyclic voltammetry scan was run with $1 \mathrm{mM} \mathrm{FeCN}$ in $1 \mathrm{M} \mathrm{KCl}$ between a potential window of $-0.1 \mathrm{~V}$ and $+0.6 \mathrm{~V}$ at a scan rate of $0.1 \mathrm{~V} \mathrm{~s}^{-1}$. The working electrode was deemed suitable if the experimental anodic peak current $\left(\mathrm{i}_{p}\right)$ of FeCN was within $5 \%$ of the theoretical $\mathrm{i}_{p}$ value $(1.471 \mathrm{x}$ $10^{-5} \mathrm{~A}$, based on the Randles-Sevcik equation). Electrodes that failed to meet this were subjected to further cleaning and polishing to bring their properties into line with this standard.

Prior to measurements of $5-\mathrm{HT}$, a cyclic voltammogram was firstly obtained in $0.1 \mathrm{M}$ PBS, $0.5 \% \mathrm{w} / \mathrm{v}$ mucin in PBS and $5 \% \mathrm{w} / \mathrm{v}$ albumin in PBS between a potential window of $0 \mathrm{~V}$ and $+1 \mathrm{~V}$ at a scan rate of $0.1 \mathrm{~V} \mathrm{~s}^{-1}$ in order to assess the capacitive current in these media. For measurements of 5-HT, $10 \mu \mathrm{M} 5-\mathrm{HT}$ was dissolved in the various matrices. An initial cyclic voltammogram was carried out between 0 to $0.8 \mathrm{~V}$ at a $0.1 \mathrm{~V} \mathrm{~s}^{-1}$ scan rate. Following this scan, an amperometric run was carried out at a potential of $0.6 \mathrm{~V}$ on the BDD and GC electrode for 5 minutes. Following this, another cyclic voltammogram was obtained to understand the degree of loss in the faradaic current due to the fouling that took place during the preceding amperometric run. This process repeated with cyclic voltammograms carried out after the potential had been held during the amperometric step for 5, 10, 20, 40, 60, 80 and 100 minutes.

\subsection{Data analysis}

The capacitive current, anodic peak potential $\left(E_{p a}\right)$, anodic half peak potential (Ena) and anodic current $\left(I_{p a}\right)$ were measured from the experimental data. Analysis was carried out using the $\mathrm{CHI} 630 \mathrm{~B}$ software. The current was expressed as the normalised current, which is the change in the current at a given time in comparison to the initial current obtained from the first scan, in a given matrix. The peak spread was measured for each response using the equation $\Delta E=\mid E_{p a}-E_{\text {hal }}$. Data was 
shown as mean \pm st.dev. and statistical analysis was carried out using a two-way ANOVA with an bonferroni test ad hoc.

\section{Results and Discussion}

\subsection{Background responses}

Figure $1 \mathrm{~A}$ displays the response of $5-\mathrm{HT}$ at $\mathrm{GC}$ and BDD electrodes in PBS whilst figures $1 \mathrm{~B}-\mathrm{C}$ display the background currents at these electrodes in the various different matrices. The $\mathrm{E}_{\mathrm{pa}}$ for $5-\mathrm{HT}$ is observed at similar potentials on both electrode materials ( $395 \mathrm{mV}$ on $\mathrm{GC}$ electrode and $420 \mathrm{mV}$ on BDD electrode). At this scan rate, no reversal peak was observed, suggestive of an irreversible oxidation mechanism occurring at both electrodes. However other researchers have shown that at higher scan rates $\left(>100 \mathrm{~V} \mathrm{~s}^{-1}\right)$, the cathodic peak $\left(E_{p c}\right)$ can be observed [11, 15]. 5-HT undergoes an electrochemical-chemical mechanism. Following oxidation 5-HT forms a radical that reacts with another 5-HT molecule forming dimers and trimers. These species then eventually polymerise on the electrode surface reducing the stability and sensitivity of the electrode for further measurements $[16,17]$.

The background capacitive currents for the GC and BDD electrodes are displayed in Figure 1B-C. The capacitive current in PBS for the GC electrode (54 $\pm 7 \mu \mathrm{F} \mathrm{cm}{ }^{-2}$ ) is significantly higher than the capacitive current on the BDD electrode $\left(18 \pm 7 \mu \mathrm{F} \mathrm{cm}^{-2}\right.$, $\mathrm{p}<0.001, \mathrm{n}=3$, Figure $1 \mathrm{C}$ ). Interestingly, the capacitive current is not significantly difference at the GC and BDD electrodes in the presence of albumin. However from $+0.6 \mathrm{~V}$ on the GC electrode and $+0.7 \mathrm{~V}$ on the BDD electrode there is a sharp increase in the background current observed in $0.5 \% \mathrm{w} / \mathrm{v}$ mucin in $0.1 \mathrm{M}$ PBS with an oxidative peak at $\sim 1.0 \mathrm{~V}$ occurring at the BDD electrode. The peak current also varied with increased $\% \mathrm{w} / \mathrm{v}$ mucin, suggestive that mucin itself can be oxidised on both electrodes. Mucin is a glycoprotein and thus contains various oxidizable amino acids and glucose units. The oxidation peak observed at $\sim 1.0 \mathrm{~V}$ may be due to either cysteine groups or glucose, which has been shown to be directly oxidised on the BDD electrode [18]. While this additional oxidation peak occurs at significantly different potentials to $5-\mathrm{HT}$, the background capacitance currents could pose a hindrance to performing analytical measurements of $5-\mathrm{HT}$ in $0.5 \% \mathrm{w} / \mathrm{v}$ mucin. The 
background capacitance current depends on the active surface area of the working electrode, as the working electrode is fouled by $5-\mathrm{HT}$, a decrease in the background capacitance current is observed as shown in Figures 2A-C and Figure 3A-C.

\subsection{Stability of glassy carbon electrode}

Carbon-based electrodes have been the electrode of choice for in vitro and in vivo recordings from biological tissue [19-24]. Figure 2 displays the stability of the GC electrode for the measurement of $5-\mathrm{HT}$ in various different matrices over a period of 100 minutes. Cyclic voltammograms examining the response of $10 \mu \mathrm{M} \mathrm{5-HT}$ are shown for all 3 biological matrices (Figure 2A-C). As seen from the background responses in Figure 1, a sharp increase in the background current at $\sim 0.5$ to $0.6 \mathrm{~V}$ is observed on all voltammograms performed in mucin. The degree of electrode fouling is significantly different in the varying matrices investigated $(p<0.001,2$-way ANOVA, $n=3$, Figure 2D). A significant difference was also observed in the current obtained with time $(p<0.001,2$-way ANOVA, $n=3$, Figure 2D). The greatest rate of fouling occurs between the initial examination and at the 5 minute mark for all electrode and matrix combinations. If the current at 5 minutes is compared to the remainder of the measurement duration, the stability of the electrode in a given matrix can be understood. These normalized currents indicate that there is no significant difference in the current response observed for $5-\mathrm{HT}$ on the GC electrode from 5 to 100 minutes in PBS or $5 \% \mathrm{w} / \mathrm{v}$ albumin (Figure 2D).

When looking at the normalized current in Figure 2D, the mucin environment increases the rate of $5-\mathrm{HT}$ fouling on the GC electrode in comparison to the other matrices. The current response for $5-\mathrm{HT}$ is completely suppressed after 40 minutes of exposure. This fouling occurs to a lesser extent in PBS with the GC electrode losing $87.5 \pm 9.2 \%$ of the initial current after 100 minutes. In contrast, $5 \% \mathrm{w} / \mathrm{v}$ albumin in $0.1 \mathrm{M}$ PBS provides the best medium for long-term measurements with the GC electrode, displaying a $75.0 \pm 12.7 \%$ current loss after 100 minutes of exposure. At the 100 minute mark, there is a significant increase in the normalized current response when measurements are carried out in $0.5 \% \mathrm{w} / \mathrm{v}$ albumin in comparison to PBS buffer $(p<0.05$, bonferroni test, Figure 2D). Although there significant fouling, it appears that the albumin matrix provides some protection to the 
electrode surface, limiting the rate of fouling in the presence of $5-\mathrm{HT}$. This may be due to a potential EC-type mechanism where 5-HT radicals may interact with albumin, preventing electrode fouling from the polymer produced through byproducts of 5-HT oxidation. These responses are interesting as the degree of fouling varies significantly between the different matrices. This is of particular interest as there is an assumption that bio-fouling from proteins will behave in a similar fashion to alter the electrode response regardless of the matrix. However albumin and mucin, two different proteins, vastly influence the rate of $5-\mathrm{HT}$ fouling on the GC electrode, where one protein performs a protective role while the other enhances the degree of fouling.

When observing the $E_{p a}$, there was a significant difference in the $E_{p a}$ values between the matrices $(p<0.001, n=3$, Figure $2 E)$ and there was a general trend of $E_{p a}$ increasing with exposure time. This is suggestive of a decreased electrode kinetics over time and is again supportive of electrode fouling hindering the response of 5-HT to different extents in different matrices. Interestingly, there was a significant difference in the $E_{p a}$ for $5-H T$ in the PBS buffer $(p<0.001, n=3)$ compared to $\% \% w / v$ albumin. This would suggest that a greater overpotential is required for the oxidation of $5-\mathrm{HT}$ in albumin compared to PBS. This may be due to the interaction of the electrode surface with albumin and/or due to the interaction of 5-HT with albumin. While the $5-\mathrm{HT}$ response in $0.5 \% \mathrm{w} / \mathrm{v}$ mucin in $0.1 \mathrm{M}$ PBS as initially similar to that occurring in PBS it had the steepest increase in Epa with time approaching similar potentials required for the oxidation of $5-\mathrm{HT}$ in $5 \%$ albumin in $0.1 \mathrm{M}$ PBS after $\sim 40$ minutes of exposure.

There was no significant difference observed in the peak spread over time in all three matrices due to the large variation observed under the three matrices (Figure 2F). In conclusion, using a GC electrode for the long-term measurements of $10 \mu \mathrm{M}$ $5-\mathrm{HT}$ displayed the most stable responses in $5 \% \mathrm{w} / \mathrm{v}$ albumin in $0.1 \mathrm{M}$ PBS in comparison to studies performed in PBS or $0.5 \% \mathrm{w} / \mathrm{v}$ mucin in $0.1 \mathrm{M}$ PBS. In contrast, using a GC electrode in $0.5 \% \mathrm{w} / \mathrm{v}$ mucin gave the poorest stability with the response due to $5-\mathrm{HT}$ being suppressed before 60 minutes of exposure during amperometry was completed. 


\subsection{Stability of boron-doped diamond electrode}

Figure 3 displays the stability of the BDD electrode for the measurement of $5-\mathrm{HT}$ over a period of 100 minutes in the three different matrices. Cyclic voltammograms for the response of $10 \mu \mathrm{M} \mathrm{5-HT}$, initially and at 10, 20 60 and 100 minute intervals are shown for all 3 biological matrices (Figure $3 \mathrm{~A}-\mathrm{C}$ ). A greater overpotential and varying peak shape is observed in the presence of $0.5 \% \mathrm{w} / \mathrm{v}$ mucin and $5 \% \mathrm{w} / \mathrm{v}$ albumin. This is due to sluggish electron transfer and potentially hindered diffusion to the electrode surface. As seen from the background responses in Figure 1C, a sharp increase in the current is observed on all voltammograms performed in $0.5 \% \mathrm{w} / \mathrm{v}$ mucin in $0.1 \mathrm{M}$ PBS at $\sim 0.5-0.6 \mathrm{~V}$. There was significant difference in the degree of fouling and the corresponding current response for $5-\mathrm{HT}$ in each matrix $(\mathrm{p}<0.001,2-$ way ANOVA, $n=3$, Figure 3D). A significant difference was observed in the current response with time $(p<0.001,2$-way ANOVA, $n=3$, Figure $3 D)$. In addition, there was also a significant difference in the rate of fouling by $5-\mathrm{HT}$ between the various matrices on the BDD electrode ( $p<0.01$, 2-way ANOVA, $n=3$, Figure 3D). The most extensive degree of fouling occurs between the initial recording and at the 5 minute mark. If the current responses for the later samples are compared back to the 5 minute mark, the stability of the electrode within a given matrix can be studied. There was no significant difference in the normalized current on the BDD electrode from 5 to 100 minutes in either PBS or $5 \% \mathrm{w} / \mathrm{v}$ albumin.

When looking at the normalized current in Figure 3D, the mucin environment caused a rapid rate of fouling by $5-\mathrm{HT}$ on the BDD electrode in a similar manner to that observed on the GC electrode. The normalized current response was significantly lower in $0.5 \% \mathrm{w} / \mathrm{v}$ mucin in $0.1 \mathrm{M}$ PBS when compared to all other matrices at all times ( $p<0.01$, bonferroni test). The current is completely suppressed after 40 minutes exposure. In PBS, the BDD electrode loses $49.4 \pm 12.7 \%$ of initial current after 100 minutes. However, unlike the GC electrode, the degree of fouling in albumin is similar to that occurring in PBS, where after 100 minutes of exposure 50.7 $\pm 1.9 \%$ of the current was lost. The degree of $5-\mathrm{HT}$ fouling in PBS and albumin was significantly greater on the GC electrode in comparison to the BDD electrode $(p<0.01, n=3)$. However there was a significant decrease in the response of $5-\mathrm{HT}$ in albumin at 40 minutes $(p<0.01, n=3$ Fig $3 D)$ and 60 minutes $(p<0.01, n=3)$ when compared to PBS. However the response recovered following 40 minutes to similar 
values to that observed in PBS. This may be suggestive that while biofouling of the BDD surface in the presence of albumin was occurring the interaction of the protein to the electrode surface may be weak, resulting on the protein film detaching from the electrode surface after passing a critical mass. A similar process was explained for a study measuring dopamine fouling [25]. As for the GC electrode, the degree and rate of fouling of the BDD electrode's surface by $5-\mathrm{HT}$ is altered in the presence of various matrices.

Overall, given the variance observed for the normalized current and the suggestion that the film is capable of detaching from the electrode surface, the rate at which the electrode surface becomes inactive due to fouling could be thought of as two separate processes. The first is the rate of formation of the 5 -HT-based polymer as $5-\mathrm{HT}$ is oxidized. The second is the removal of this polymer from the surface either from failing to adhere to the electrode surface or growing to a size that detaches the film from the electrode. In this, the degree to which the electrode surface is no longer active is a combination of these two processes. This would explain the seemingly more random variance observed for the normalized current for 5-HT with exposure for both the GC and BDD electrodes in PBS and albumin, particularly at the BDD electrode, as these processes compete with one another. In addition, it would also be possible that the rate of fouling by the 5 -HT-based polymer may be functionally identical in each matrix at each electrode, with the stability of this film on the surface giving rise to the observed differences in the rate at which the electrode surface is rendered inactive. Given that the normalized current for oxidation of 5-HT in mucin at both the GC and BDD electrodes display reduced variance, in comparison to measurements in PBS and albumin, would suggest that the 5-HT-based polymer film on the working electrode is more stable with more predictable growth in the mucin matrix. This would correspond to the increased rate of fouling in a mucin matrix relative to the PBS and albumin matrices.

When examining the $\mathrm{E}_{\mathrm{pa}}$ of $5-\mathrm{HT}$, there was a significant difference between $\mathrm{E}_{\mathrm{pa}}$ responses between the matrices $(p<0.001, n=3$, Figure $3 E)$ with a general trend of $E_{p a}$ increasing over time. This is suggestive of increasingly poor kinetic behaviour and is indicative of electrode fouling. This behaviour was similar to that observed at the GC electrode. Once again the response for $5-\mathrm{HT}$ in mucin had the steepest increase in $E_{p a}$ with time while the response for the $E_{p a}$ of $5-H T$ in PBS and albumin 
was relatively stable in comparison. Once again a greater $\mathrm{E}_{\mathrm{pa}}$ was required for the oxidation of $5-H T$ in albumin in comparison to PBS $(p<0.001, n=3)$. There was a significant difference observed in the peak spread over time in all three matrices (Figure $3 \mathrm{~F}$ ) on the BDD electrode. Although there was no significant difference in peak spread over time, there was a general increase. In conclusion, there is no difference between PBS and albumin matrices for the long-term measurements of 10 $\mu \mathrm{M} 5$ - HT with a BDD electrode. A mucin environment gave the highest rate of electrode fouling, with the current response due to $5-\mathrm{HT}$ being completely suppressed within 40-60 minutes of exposure.

\section{Conclusion}

Measurements in various matrices are becoming more common with the widely applicability of biological monitoring. Overall this study suggests that all biological matrices cannot be treated in a similar fashion even if the biological elements within a given matrix are potentially similar. For the continuous monitoring of 5-HT using a GC electrode, $5 \% \mathrm{w} / \mathrm{v}$ albumin in PBS was shown to act in a protective fashion, whilst $0.5 \% \mathrm{w} / \mathrm{v}$ mucin in PBS was detrimental to the observed response. However using BDD electrode to monitor $5-\mathrm{HT}$ the presence of the two different proteins in albumin and mucin did not alter the degree of electrode fouling. Other than the mucin matrix, the BDD electrode was more stable in all other matrices compared to the GC electrode.

\section{Acknowledgements}

AFM and BAP would like to thank EPSRC (EP/J000175/1) for funding.

\section{References}

[1] D. Baldwin and S. Rudge, International Clinical Psychopharmacology $\underline{9}: 41$ (1995).

[2] M. D. Gershon, Advances in Experimental Medicine:221 (1991).

[3] S. Ge, E. Woo, J. G. White, and C. L. Haynes, Analytical Chemistry 83:2598 (2011). 
[4] S. R. Jones, G. E. Mickelson, L. B. Collins, K. T. Kawagoe, and R. Mark Wightman, Journal of Neuroscience Methods $\underline{52: 1}$ (1994).

[5] B. T. Chen and M. E. Rice, Electroanalysis 11:344 (1999).

[6] G. Marcelli and B. A. Patel, Analyst 135:2340 (2010).

[7] B. A. Patel, The Analyst 133:516 (2008).

[8] M. Frost and M. E. Meyerhoff, Analytical Chemistry 78:7370 (2006).

[9] P. P. Bertrand, J Physiol 577:689 (2006).

[10] R. Kachoosangi and R. Compton, Analytical and Bioanalytical Chemistry 387:2793 (2007).

[11] B. P. Jackson, S. M. Dietz, and R. M. Wightman, Analytical Chemistry 67:1115 (1995).

[12] B. A. Patel, X. Bian, V. Quaiserova-Mocko, J. J. Galligan, and G. M. Swain, The Analyst 132:41 (2007).

[13] B. E. K. Swamy and B. J. Venton, The Analyst 132:876 (2007).

[14] A. G. Guell, K. E. Meadows, P. R. Unwin, and J. V. Macpherson, Physical Chemistry Chemical Physics 12:10108 (2010).

[15] P. Hashemi, E. C. Dankoski, J. Petrovic, R. B. Keithley, and R. M. Wightman, Analytical Chemistry 81:9462 (2009).

[16] G. Dryhurst, Chemistry Reviews 90:795 (1990).

[17] M. Z. Wrona and G. Dryhurst, The Journal of Organic Chemistry $\underline{52: 2817}$ (1987).

[18] J. Lee and S.-M. Park, Analytica Chimica Acta 545:27 (2005).

[19] J. Park, V. Quaiserova-Mocko, B. A. Patel, M. Novotny, A. Liu, X. Bian, J. J. Galligan, and G. M. Swain, The Analyst 133:17 (2008).

[20] B. A. Patel, Neurogastroenterology \& Motility 23:595 (2011).

[21] K. L. Adams, M. Puchades, and A. G. Ewing, Annual Review of Analytical Chemistry (2008) 1 (2008).

[22] B. W. Allen and C. A. Piantadosi, Nitric Oxide $\underline{8}: 243$ (2003).

[23] D. Bruns, Methods 33:312 (2004).

[24] K. F. Martin, C. A. Marsden, and F. Crespi, TrAC Trends in Analytical Chemistry $\underline{7}: 334$ (1988).

[25] R. Trouillon and D. O'Hare, Electrochimica Acta 55:6586 (2010). 


\section{Figure Captions}

Figure 1. Background current responses in various matrixes. Voltammograms for 50 $\mu \mathrm{M} 5-\mathrm{HT}$ is shown on the BDD and GC electrode (A) in PBS, where the anodic peak potential is similar on both electrodes. The background currents in the various matrixes are shown on the $\mathrm{GC}(\mathrm{B})$ and $\mathrm{BDD}(\mathrm{C})$ electrode. Voltammograms were run at $0.1 \mathrm{~V} \mathrm{~s}^{-1}$

Figure 2. CVs displaying the responses of $10 \mu \mathrm{M} 5-\mathrm{HT}$ the $\mathrm{GC}$ electroder recorded in a PBS buffer (A), $5 \% \mathrm{w} / \mathrm{v}$ bovine serum albumin (B) and $0.5 \% \mathrm{w} / \mathrm{v}$ porcine stomach mucin (C). Voltammograms were run at $0.1 \mathrm{~V} \mathrm{~s}^{-1}$ over a potential window of 0 to $+0.8 \mathrm{~V}$, at specified time frame exposures of $0,10,20,60$ and 100 minutes following amperometric recordings at $+0.6 \mathrm{~V}$. The normalized current (\%) response is shown in D. The anodic peak potential $\left(E_{p a}\right)$ and the peak spread $\left(E_{p a}-E_{p h}\right)$ are shown in $E$ and $F$ respectively. Data is shown as Mean \pm st. dev., ${ }^{*} p<0.05$ PBS buffer vs. albumin response.

Figure 3. CVs displaying the responses for $10 \mu \mathrm{M} 5-\mathrm{HT}$ at the BDD electrode recorded in PBS buffer (A), $5 \% \mathrm{w} / \mathrm{v}$ bovine serum albumin (B) and $0.5 \% \mathrm{w} / \mathrm{v}$ porcine stomach mucin (C). Voltammograms were run at $0.1 \mathrm{~V} \mathrm{~s}^{-1}$ over a potential window of 0 to $+0.8 \mathrm{~V}$, at specified time frame exposures of $0,10,20,60$ and 100 minutes following amperometric recordings at $+0.6 \mathrm{~V}$. The normalized current (\%) response is shown in D. The anodic peak potential (Epa) and the peak spread (Epa - Eph) are shown in $E$ and $F$ respectively. Data is shown as Mean \pm st. dev., ${ }^{*} p<0.05$ and ${ }^{* *} p<0.01$ PBS buffer vs. albumin response. 
FIGURE 1
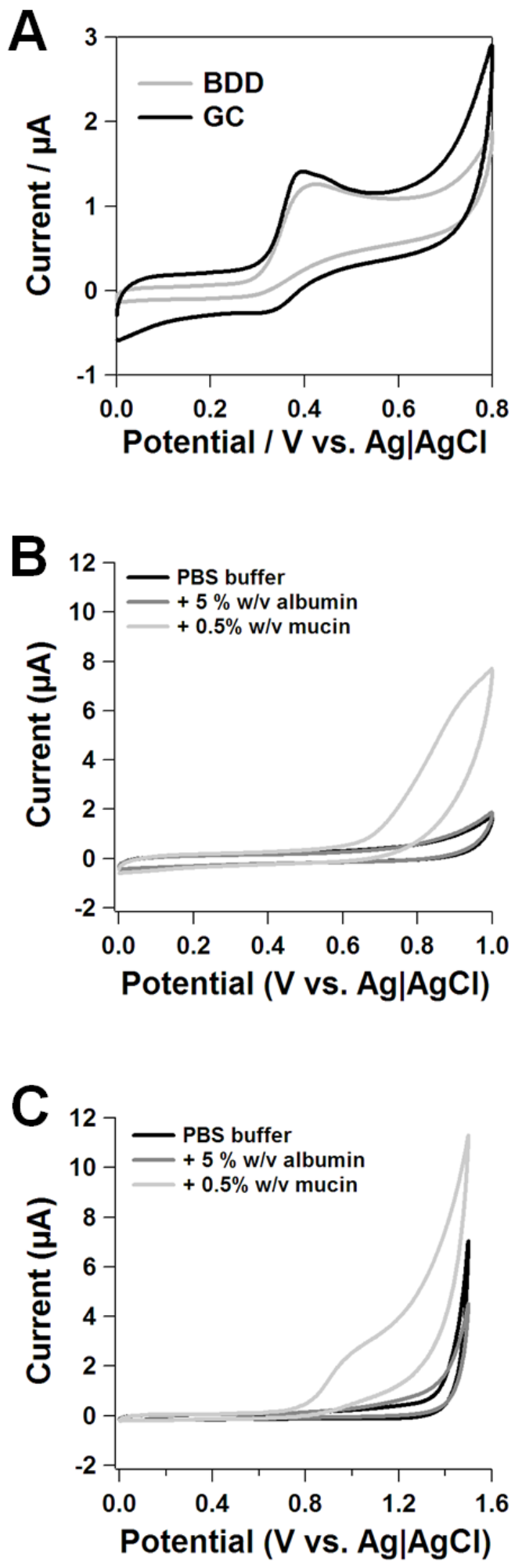


\section{FIGURE 2}
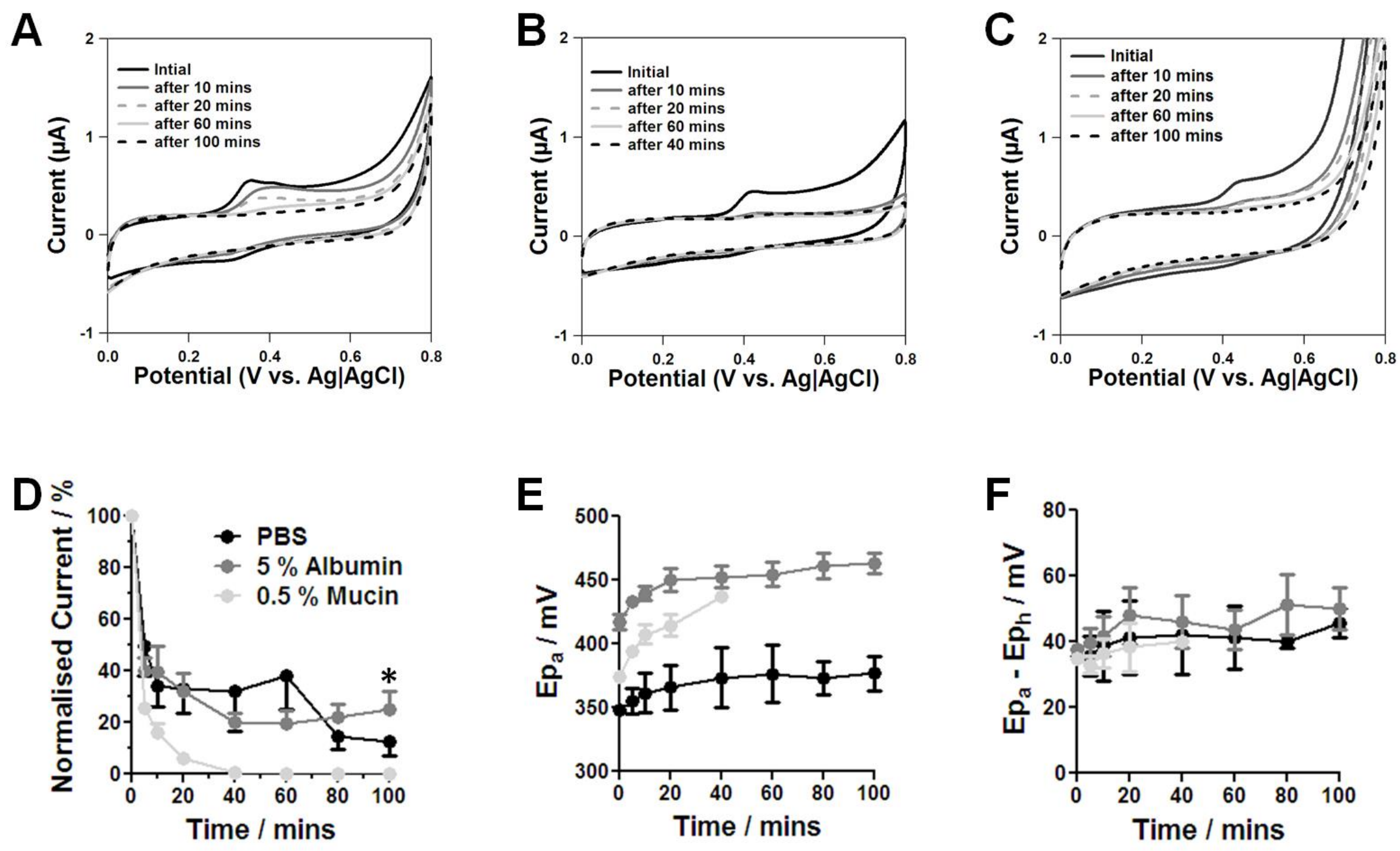


\section{FIGURE 3}
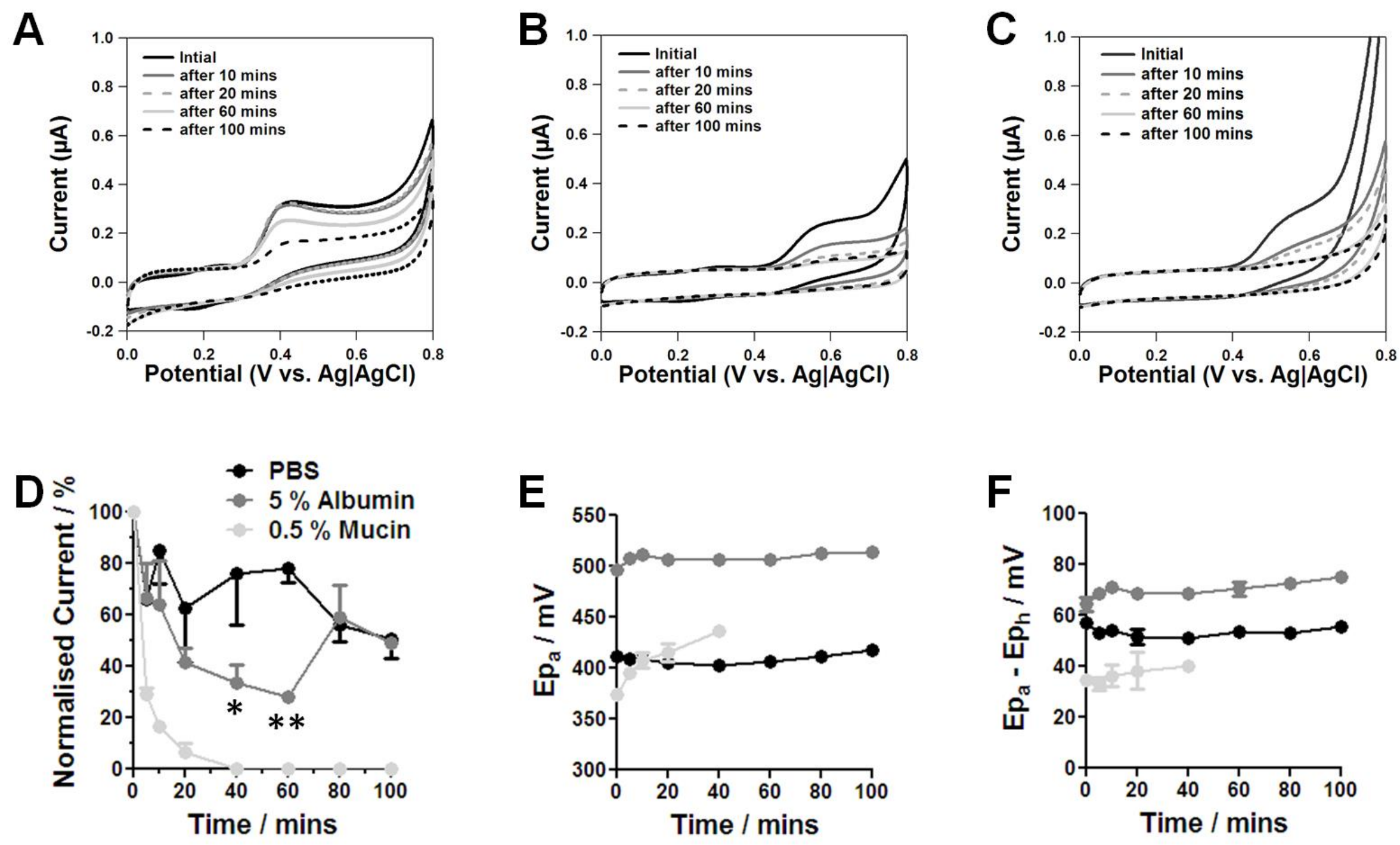
